\title{
Pour l'utilisation des SIG (systèmes d'information géographique) en histoire des techniques : entre documentation et analyse spatiale
}

For the use of GIS (geographical information systems) in technical history:

between documentation and spatial analysis

Jean-Louis Kerouanton

\section{OpenEdition \\ Journals}

Édition électronique

URL : http://journals.openedition.org/dht/299

DOI : 10.4000/dht.299

ISSN : $1775-4194$

Éditeur :

Centre d'histoire des techniques et de l'environnement du Cnam (CDHTE-Cnam), Société des élèves du CDHTE-Cnam

\section{Édition imprimée}

Date de publication : 1 décembre 2009

Pagination : 81-94

ISBN : 978-2-9530779-4-0

ISSN : 0417-8726

Référence électronique

Jean-Louis Kerouanton, «Pour l'utilisation des SIG (systèmes d'information géographique) en histoire des techniques : entre documentation et analyse spatiale ", Documents pour l'histoire des techniques [En ligne], 18 | $2^{\mathrm{e}}$ semestre 2009, mis en ligne le 24 septembre 2010, consulté le 08 septembre 2020 URL : http://journals.openedition.org/dht/299 ; DOI : https://doi.org/10.4000/dht.299 


\title{
Pour l'utilisation des SIG (systèmes d'information géographique) en histoire des techniques : entre documentation et analyse spatiale
}

\author{
Jean-Louis Kerouanton \\ Université de Nantes \\ Centre François Viète (EA 1161)
}

\begin{abstract}
Résumé
La recherche en histoire des techniques peut-elle passer par l'analyse spatiale? Un bilan sommaire de la question de la représentation graphique spatiale dans la littérature française permet d'ouvrir le débat sur les échelles de lecture nécessaires pour aborder la notion de paysage industriel. Les travaux en cours du Centre François Viète de l'université de Nantes se développent autour de deux approches complémentaires: les mondes portuaires et l'introduction des technologies numériques dans l'histoire. Les approches de rétroconception sont à mettre en perspective avec leur intégration dans la dimension spatiale des procédés, qui passe par le développement des SIG. L'article aborde les exemples de La Ciotat et Nantes.
\end{abstract}

Résumés et mots clés en anglais sont regroupés en fin de volume, accompagnés des mots clés français

« La carte n'est pas une réalité $»$.

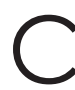
ette phrase peut paraître évidente ; elle indique cependant la nécessaire prudence que nous devons toujours avoir devant les représentations quelles qu'elles soient et tout particulièrement devant une carte ou un plan. La topographie, c'est bien connu, ne prend sens que couchée sur le papier ou lisible sur un écran d'ordinateur. Pour passer de la sphère terrestre à la lecture bidimensionnelle, il s'agit d'utiliser des systèmes de projection dont on sait qu'ils sont imparfaits's. Les lignes qui suivent traitent d'un type particulier de représentation qui concerne l'interprétation des lieux, un type de représentation fort ancien mais dont les nouveaux

1 Hervé Le Bras et Emmanuel Todd, L'invention de la France, Paris, Librairie générale française, 1981, p. 89.

2 C'est par exemple la comparaison classique entre la projection ancienne de Mercator et celle de Peters dans les années 1970, avec son équateur au milieu et son égalité des surfaces représentées qui a remis en cause une lecture « nordiste » du monde. outils numériques autorisent depuis bientôt deux décennies la manipulation par un nombre croissant de chercheurs et d'étudiants. Plus récemment encore, la généralisation des outils personnels de navigation de type GPS, les tracés d'itinéraires et l'accès aux cartes routières ou aux plans de ville sur internet nous familiarisent avec un certain type de cartographie utilisant les systèmes d'information géographique (SIG). Nous tenterons d'abord une revue sommaire des représentations spatiales en histoire des techniques ${ }^{3}$. Les cas qui seront présentés ensuite relèvent de recherches développées depuis plusieurs années au sein de l'équipe d'histoire des techniques du Centre François Viète de l'université de Nantes ${ }^{4}$. Entre procédés industriels et patrimoine,

\footnotetext{
3 En limitant nos exemples à la France.

4 Florent Laroche, Michel Cotte, Jean-Louis Kerouanton, Alain Bernard, « Objet, société, technologies de l'information et de la communication : l'apport du patrimoine et de I'histoire des techniques dans la capitalisation des connais-
} 
ils se situent en totale continuité avec les travaux de modélisation qu'évoquent Michel Cotte et Florent Laroche dans leurs articles respectifs de ce numéro des Documents pour I'histoire des techniques. ${ }^{5}$. Ils émanent également, pour certains d'entre eux, d'expériences précédentes au sein du ministère de la Culture et tout particulièrement dans le cadre des travaux de l'Inventaire général ${ }^{6}$. Les recherches présentées permettront nous l'espérons, d'ouvrir le débat. II ne s'agira pas dans ce court article de

sances, la valorisation et les approches méthodologiques de I'ingénieur $11,131^{\text {e }}$ congrès national des sociétés historiques et scientifiques, "Tradition et innovation 》, Grenoble, 2006, thème 8 " L'actualité du passé » (édition électronique à paraître) ; Florent Laroche, Michel Cotte, Jean-Louis Kerouanton, Alain Bernard, "L''image virtuelle comme source de connaissance pour le patrimoine technique et industriel : comment allier histoire et ingénierie ? ", dans Bertrand Lavédrine dir., Genres et usages de la photographie, $132^{\mathrm{e}}$ congrès national des sociétés historiques et scientifiques (Arles, 2007), Paris, CTHS, 2009, pp. 53-64 (édition électronique).

5 Le présent article est le prolongement et le développement d'une communication orale, "Des échelles, de l'objet, de I'espace : SIG, TIC et Histoire des techniques ॥, donnée dans le cadre du Congrès de la Société française d'histoire des sciences et des techniques, Paris, 3-5 septembre 2008, Session Histoire des techniques. Cette réflexion n'existerait pas sans l'apport de certains collègues depuis plusieurs années déjà, tout particulièrement Christophe Batardy du ministère de la Culture dont nous citons l'article écrit au moment où il nous faisait partiellement découvrir les SIG : « La cartographie automatique et les systèmes d'information géographique, aperçu méthodologique ", Jean Steinberg dir., Cartographie pratique pour la géographie et l'aménagement, Paris, SEDES, 1996, pp. 111-121.

6 Jean-Lovis Kerouanton, Daniel Sicard, collab. Association d'histoire de la construction navale à Nantes, Alain Bouras, Michel Pacault, La construction navale en Basse-Loire, Loire-Atlantique, Images du patrimoine, Inventaire général, Nantes, Association pour le développement de l'Inventaire général des Pays de la Loire (ADIG), 1992 ; Jean-Louis Kerouanton dir., LU : une usine à Nantes, Images du Patrimoine, Inventaire Général, Nantes, ADIG, 1999 (édition revue et augmentée); id. dir., dossier « Nantes : un modèle ? ॥, $L^{\prime}$ archéologie industrielle en France, $n^{\circ} 41$, décembre 2002 , pp. 4-93 ; id., «Usines des îles, usines de Loire : cas de figures à Nantes 1 , ibid., pp. 14-23. Pour ce qui est de la cartographie proprement dite : Jean-Lovis Kerouanton, « La tabletterie et la brosserie en France, 1845-1936. Matériaux statistiques ", dans Bertrand Fournier et Hélène Frichet-Colzy dir., Le bois, l'os, la corne, l'ivoire, la nacre. Aspects de la tabletterie en France, Amiens, Agir-Pic, 2001, pp. 29-40 ; id., " Éléments de cartographie pour la connaissance de l'architecture balnéaire bauloise "I, dans Les réseaux de la villégiature, In Situ, revue internet de l'inventaire général (www.revue.inventaire. culture.gouv.fr) n4, mars 2004 donner des conclusions définitives mais de réfléchir à quelques pistes provisoires en ce qui concerne l'utilisation des systèmes d'information géographiques par un historien des techniques.

Question d'échelle. La carte, de l'atelier au territoire Il faut en préambule souligner avec Jean-Luc Arnaud' le peu d'outils de travail satisfaisants pour les historiens en matière de cartographie. Si « les manuels relatifs à l'analyse spatiale sont assez nombreux " pour les architectes ou les géographes, les historiens, quand ils s'en servent n'y trouvent pas leur compte, confrontés à des procédés «souvent trop sophistiqués» alors que leurs données sont lacunaires. J.-L. Arnaud signale cependant l'opportunité nouvelle que représente l'évolution des techniques informatiques facilitant la manipulation de certains outils ${ }^{8}$. Sans doute l'auteur se concentre-t-il plutôt sur les questions d' histoire urbaine mais sa réflexion toute récente (publiée au printemps 2008) sur l'analyse spatiale et la cartographie en fait l'une des références incontournables'. Nous renverrons également à une publication de 2004 de la revue Histoire et Mesure plus spécifiquement dévolue aux systèmes d'information géographique qui fait la part belle aux problématiques de cartographie et d'espace, appliquées à l'archéologie et l'urbanisme 10 .

Avant de tenter quelques entrées vers l'histoire des techniques, nous utiliserons ces deux références pour donner les définitions générales et les nuances préliminaires qui semblent indispensables:

"L'analyse spatiale est un ensemble de démarches qui visent à décrire l'organisation des structures matérielles de l'espace et les manières dont il est occupé. Ces démarches sont surtout mises en œuvre par les géographes, les architectes, les anthropologues et les sociologues. Elles intéressent cependant les historiens dès qu'ils ne considèrent pas l'espace comme une toile de fond uniforme et sans qualification, comme un simple lieu

7 Jean-Luc Arnaud, Analyse spatiale, cartographie et histoire urbaine, Marseille/Aix-en-Provence, Parenthèses/MMSH, 2008. 8 lbid., pp. 9-10. Nous nous garderons bien d'évoquer les logiciels utilisables aujourd'hui, tant l'offre est évolutive, tout particulièrement en ce qui concerne les logiciels libres. Une source internet générale cependant : www.portailsig.org.

9 Le constat de Jean-Luc Arnaud est à confronter avec les propos comparables tenus voilà pourtant plus de vingt ans dans son avant-propos par Xavier de Planhol, Géographie historique de la France, Paris, Fayard, 1988, pp. 9-10.

10 Dossier « Systèmes d'information géographique, archéologie et histoire ॥, Histoire et Mesure, vol. XIX, n³/4, 2004 
privilégié d'observation. L'utilisation de l'analyse spatiale en histoire repose sur deux hypothèses quant à la manière dont l'espace se constitue : 1) les différences de distribution d'un phénomène ne résulte pas du hasard ; elles en sont l'expression concrète ; 2) la distribution des phénomènes dans l'espace n'est pas neutre dans la manière dont ils évolvent et se transforment $\|^{11}$.

« D'une façon générale, la conception d'un SIG est avant tout un processus de modélisation d'une problématique intégrant une dimension spatiale ou géographique, de structuration des phénomènes retenus comme caractérisant, concrétisant celle-ci avant d'être un problème d'ordre informatique. Ces phénomènes sont à l'intersection de trois espaces ou ensembles, à savoir l'espace de la réalité observable (thématique, sémantique), l'espace spatial ou géographique et l'espace temporel. L'information spatiale ou géographique créée correspond à une des parties visibles des phénomènes et n'a de sens que rapportée à ces espaces pris ensemble $\aleph^{12}$.

Pour Françoise Pirot et Anne Varet-Vitu en effet, « créer un SIG est une démarche intellectuelle reposant sur une approche systémique alors que la création d'information spatiale matérialise uniquement la structure des objets géographiques "I; il ne s'agit donc pas seulement de gérer les données pour les organiser facilement sur une carte grâce à une simple manipulation informatique.

II n'est pas sûr que ce nous présentons dans cet article dépasse le stade de la simple compilation documentaire pour arriver au statut véritable de système d'information géographique tel que défini à l'instant. Conscient que nous n'empruntons à Nantes guère plus qu'un chemin, l'enjeu véritable de notre travail est de proposer les prémisses d'une analyse qui permette d'engager la connaissance des objets techniques selon leurs emplacements respectifs, bref d'en faire une réelle analyse spatiale. Mais pas d'analyse spatiale sans organisation de la connaissance sous forme cartographique. L'idée que nous défendons est d'essayer à terme le passage de l'objet technique (ou/et industriel) à son territoire, à travers l'analyse des « process », le lien de machine à machine dans le fil de la production lui-même, de son environnement immédiat, l'atelier ou l'usine, à son territoire de production et d'usage. En somme,

11 J.-L. Arnaud, Analyse spatiale, op.cit., p. 7.

12 Françoise Pirot et Anne Varet-Vitu, « Introduction », Dossier "Systèmes d'information géographique, archéologie et histoire "), Histoire et Mesure, vol. XIX, n॰3/4, 2004, pp. 219-222. le territoire pris depuis l'amont dans sa logique de fabrication jusqu'à l'aval par ses usages ou sa logique d'écoulement marchand ${ }^{13}$. C'est dire que la notion d'échelle paraît extrêmement importante : on pourrait ainsi étudier la machine dans son atelier ou/et passer à l'échelle régionale de la production (par exemple la construction navale dans le cas de l'industrie) ou de l'utilisation (ainsi les navires, dans celui des transports). En terme de cartographie, il ne s'agit donc plus seulement de considérer comme carte la carte régionale ou nationale mais également le plan de la ville, du quartier, de l'usine jusqu'à celui de l'atelier dans lequel se joue le cœur du procédé technique avec ses machines, ses outils, ses gestes et ses pratiques.

À ce titre, l'intégration des nouvelles technologies et la confrontation avec la question de la modélisation ou de la conception assistée par ordinateur ouvrent de nouvelles perspectives complémentaires pour la compréhension de l'objet technique. Parlant à la fois d'objet et d'espace, on voit bien comment la recherche s'inscrit désormais dans une interrogation sur la question des échelles (des temps, des territoires) où le geste, le savoir-faire, le procédé composent un système sociotechnique.

\section{Y a-t-il une faiblesse de la cartographie en histoire des techniques?}

Sans avoir la prétention de faire un bilan exhaustif, il ne semble pas inutile de revenir sur certaines publications plus ou moins anciennes pour tenter d'en dresser un panorama rapide et en marquer quelques évolutions.

L'histoire des techniques passe notamment par la présentation des machines, très régulièrement par les coupes des objets et plus rarement des installations immédiatement environnantes, également très généralement en coupes : nombreuses sont bien sûr les illustrations en ce sens dans les irremplaçables ouvrages pionniers de Bertrand Gille et de Maurice Daumas ${ }^{14}$. Mais il ressort immédiatement que le passage au plan de l'atelier relève de l'exception. De telles figures se comptent à peine sur les doigts d'une main dans chaque volume de l'Histoire générale de Maurice Daumas ${ }^{15}$.

13 On comprendra sans peine ce que nous devons comme tant d'autres à la question des systèmes techniques à propos desquels il suffira ici de citer Yves Deforge, Technologie et génétique de l'objetindustriel, Paris, Maloine, 1985 et bien entendu Bertrand Gille, Histoire des techniques, Paris, Gallimard, 1978. 14 Maurice Daumas dir., Histoire générale des techniques, Paris, PUF, 5 vol. 1962-1979.

$15 \mathrm{lbid}$. En dehors des quelques plans d'urbanisme, citons les quelques exemples liés au discours technique : dans le 
Deux plans d'usine chez M. Daumas ${ }^{16}$ mais pas plus dans l'Histoire des techniques de B. Gille ${ }^{17}$ dans laquelle pourtant le deuxième plan est bien censé expliciter les nouvelles organisations de l'industrie automobile contemporaine. Le discours sur l'espace technique est évidemment présent dès ces ouvrages fondateurs mais force est de constater que la traduction graphique en plan ou en carte, illustrative ou analytique est globalement absente.

C'est peut-être par le biais des approches patrimoniales ettoutparticulièrement del'archéologie industrielle que le mouvement d'analyse spatiale en histoire des techniques a connu en France de nouveaux développements. Accompagnant à la fin des années 1970 la naissance et le développement d'une importante association militante en faveur du patrimoine industriel, le Cilac, la publication par Maurice Daumas de son Archéologie industrielle en France marque incontestablement un tournant ${ }^{18}$. Plaçant l'usine dans son contexte physique et non plus seulement purement économique, Maurice Daumas mettait ainsi délibérément sa longue expérience d'historien des techniques (et avec lui, celle du Centre d'histoire des techniques du Cnam) au service de la compréhension du "paysage industriel " dont il précisait dans des chapitres importants, la définition générale et ses " éléments singuliers $11^{19}$. L'attention aux développements matériels bâtis des usines anciennes réclamait ainsi naturellement une représentation graphique bien plus systématique des objets techniques de production dans leur environnement. Sur les presque trois cents figures du livre, on peut en effet recenser, en lien étroit avec les nombreuses photographies de

volume 2, la carte des chemins de Saint-Jacques de Compostelle, p. 356, les agrandissements successifs de Toulon entre 1515 et 1701 1, p. 420 (trois plans non orientés et à des échelles différentes), les bassins de Portsmouth (avec schéma et plan des machines), p. 427 ; dans le volume 3, pour les transports modernes, rien pour la route mais la carte du Grand Trunk Canal en Angleterre, p. 300, pour les chemins de fer, les installations de voies ferrées au Creusot en 1785, p. 373 et le développement des chemins de fer en France, pp. 408-409 (quatre cartes chronologiques) ; seulement deux plans d'établissement industriel, dans le volume 4 enfin, dont la carte des usines électriques de Paris, p. 443.

16 ibid., vol. 3 : une usine de blanchissage vers 1830, p. 634 , et une sucrerie de betteraves vers 1835, p. 642.

17 B. Gille, op. cit. L'ensemble des roves hydrauliques de Tharsis, p. 397 et le plan de la nouvelle usine Volvo de Kalmar en Suède, p. 1293.

18 Maurice Daumas, L'archéologie industrielle en France, Paris, Robert Laffont, 1980

19 ibid., pp. 13-91. sites, une vingtaine de plans d'établissement ou de cartes de situations ${ }^{20}$ ce qui, compte tenu du nombre finalement limité d'exemples étudiés, constitue une proportion réellement significative. En accordant une réelle importance à l'étude des « plans de différentes époques ", la démarche de l'équipe de Maurice Daumas se voulait alors résolument pluridisciplinaire ${ }^{21}$. Les contacts avec les archéologues mais peut-être aussi avec certains chercheurs de l'Inventaire général, alors encore en plein développement, permettaient aussi des confrontations sur l'étude monumentale et ses méthodes dans un champ nouveau 22 .

Dresser un bilan de la situation actuelle, ce serait alors logiquement constater les développements réguliers d'une telle pratique. Les nouveaux outils numériques aidant, surtout à partir des années 1990 avec la généralisation des ordinateurs personnels, on constaterait que cette tendance à l'intégration territoriale et à l'analyse spatiale des phénomènes pouvait trouver naturellement sa place dans l'ensemble des études en histoire des techniques consacrées à tel ou tel développement sur un territoire donné. Mais on aura compris, dès les remarques citées plus haut de Jean-Luc Arnaud, qu'ici comme ailleurs la problématique historienne ne s'est en fait globalement pas encore emparée de la méthode et des approches spatiales, moins encore de celles de type SIG qui, pourtant, comme nous le verrons, font surface de temps à autre.

Loin de nous cependant l'idée de réduire nécessairement la discipline à telle approche plutô† qu'une autre. La technique n'est pas que territoire, loin s'en faut ${ }^{23}$. Pour comprendre, par exemple chez les Seguin, les développements des ponts suspendus ou des chaudières à vapeur, point n'est besoin pour Michel Cotte d'utiliser systématiquement la réflexion sur le territoire. La compréhension du phénomène passe plutôt par l'analyse fine des forces pour les uns ou de la première chaudière de Stephenson pour les

20 Dus à Gérard Jigaudon, ibid., p. 463. II est clair que pour bon nombre de travaux, quand cela est possible, la collaboration avec des graphistes, des infographistes et des cartographes est une plus-value incontestable.

21 lbid., p. 435.

22 lbid., p. 9.

23 Même si la dimension bâtie n'en est pas totalement exclue, nous n'avions, par exemple, pas présenté les cartes SIG des anciennes fonderies de l'Atlantique dans un article plus centré sur l'élaboration même de l'objet industriel, « De la théorie au modèle : les hélices comme sculptures calculées, le cas des fonderies de l'Atlantique à Nantes", dans Patrimoine Scientifique, In-Situ. Revue des patrimoines, $n^{\circ} 10$, mai 2009, www.revue.inventaire.cultur.gouv.fr 
autres ${ }^{24}$. Pas de cartographie en l'occurrence et c'est somme toute, normal. Le même auteur ne manque cependant pas d'établir les cartes générales du réseau fluvial rhodanien, du basin houiller de la Loire et bien entendu celle du chemin de fer de Saint-Étienne à Lyon ${ }^{25}$. Tout au plus pourrait-on lui demander à quoi pouvaient bien ressembler les ateliers de Saint-Marc à l'origine de l'entreprise ${ }^{26}$.

Un exemple parmi tant d'autres possibles mais qui pose la question des principes de l'analyse et plus spécialement des objets et des échelles considérées. Il est probable en effet que ce que nous défendons ici relève plus facilement de l'étude, non pas d'un phénomène technique général comme par exemple les développements de l'énergie à vapeur, mais de celle des paysages et des objets de production - les usines et les ateliers. C'est d'abord dans les approches monographiques sur telle ou telle usine ou tel territoire que la carte et le plan pourraient trouver naturellement leur place. Une fois ces études réalisées, les compilations plus synthétiques seraient possibles, ouvrant pour le coup sur des discours plus généraux.

On en est proche avec l'étude de Florent Laroche sur Batz-sur-Mer dans laquelle les modélisations de la laverie de sel sont d'ores et déjà intégrées à l'architecture environnante proche ${ }^{27}$. Mais d'intégration à la totalité des bâtiments, point ; ce serait, pourquoi pas, l'étape suivante. Les projets de l'équipe sont bien d'associer les différentes étapes de la réflexion, la modélisation numérique des machines pouvant s'intégrer à l'analyse de l'environnement bâti et son territoire ${ }^{28}$.

24 Michel Cotte, Le choix de la révolution industrielle. Les entreprises de Marc Seguin et ses frères (1815-1835), Rennes, Presses universitaires de Rennes, 2007, pp. 193-200 et pp. 436-437. 25 Ibid., pp. 250, 352 et 402.

26 Ibid., p. 61.

27 Florent Laroche, dans le présent volume. Mais on pourrait dire la même chose d'Alain Michel (auteur également dans ce recueil) : malgré une iconographie remarquable avec de nombreux documents graphiques, il ne publiait en 2007 qu'une seule petite synthèse cartographique faisant état de I'usine Renault en 1930 : Alain P. Michel, Travail à la chaîne : Renault, 1898-1947, Boulogne-Billancourt, ETAl, 2007, p. 79. Alain Michel passera ensuite à son projet " usine $3 \mathrm{D}$ ॥ dans lequel la question de l'étude fine des bâtiments est, cette fois, évidente. Des collaborations entre équipes sont peut-être à ce titre à envisager : Jean-Lovis Kerouanton, Florent Laroche, Alain Michel, « Histoire des techniques industrielles et reconstitutions $3 \mathrm{D} \Perp$, intervention à la journée d'étude Usages des sources numériques en histoire des sciences et des techniques-ll , Paris, Cité des sciences et de l'industrie, octobre 2009.

$28 \mathrm{Ce}$ qui permettrait la validation des circulations en dehors des ateliers, comme l'a esquissé pour l'instant sommairement Florent Laroche dans sa thèse, Contribu-
Il faut cependant dire que cette cartographie des unités de production dispose de quelques antécédents notables. La multiplication actuelle des ouvrages très largement illustrés facilite grandement la publication de documents anciens ef les plans y ont une part importante ${ }^{29}$. Mais c'est autre chose de les analyser ou d'en faire la synthèse. Là encore, pas d'inventaire exhaustif mais quelques très bons exemples récents, comme ces plans des forges de Sarthe et de Mayenne ${ }^{30}$, l'état au XVe siècle de la saline royale de Salins ${ }^{31}$ ou encore les évolutions de la manufacture d'armes de Saint-Étienne ${ }^{32}$. Ces trois réalisations représentent un réel effort documentaire mené à partir de la compilation des plans anciens, de toute évidence lié à un effort graphique remarquable ${ }^{33}$. On peut lire sur ces plans différents types d'indications chronologiques, des comparaisons d'implantations spatiales générales des différents ateliers ou encore des superpositions de projets avec leur réalisation ultérieure, qui ouvrent la voie de campagnes de traitement plus systématiques pour l'analyse des bâtiments industriels.

tion à la sauvegarde des objets techniques anciens par l'archéologie industrielle avancée : proposition d'un modèle d'information de référence muséologique et d'une méthode inter-disciplinaire pour la capitalisation des connaissances du patrimoine technique et industriel, École centrale de Nantes/Université de Nantes, sous la direction de Alain Bernard et Michel Cotte, décembre 2007, pp. 312-314. 29 Pour cette question des sources : Catherine ManigandChaplain, Les sources du patrimoine industriel, Paris, Cilac, Éditions du patrimoine, 1999.

30 Jean-François Belhoste, Évelyne Robineau et alii, La métallurgie du Maine : de l'âge du fer au milieu du $X X^{e}$ siècle, Paris, Éditions du patrimoine, 2003, p. 181 (les forges de Chemiré et de Moncor, p. 263, le soufflage à air chaud à Port Brillet ou encore, p. 274, l'évolution de l'usine de Port-Brillet de 1831 à 1970). 31 Jean-François Belhoste, Christiane Roussel, Une manufacture princière au XVe siècle : la grande saline de Salins-les-Bains, Jura, site et territoire, Besançon, Association pour la promotion et le développement de l'inventaire comtois (ASPRODIC), 2006, p. 28 par exemple, la chronologie de la reconstruction au $X V$ siècle ou P. 62, les installations techniques au XVe siècle. 32 Bernard Bacher, Jean-François Brun, Éric Perrin, La manufacture d'armes de Saint-Étienne : la révolution des machines, 1850-1870, Saint-Étienne/Clermont-Ferrand, Ville de Saint-Étienne/Musée d'art et d'industrie/Un, deux... quatre éd., 2007, entre autres p. 29 (la situation du projet de 1830). 33 II est vrai aussi que les éditions en couleurs permettent une lisibilité accrue dans ce genre de publication. 


\section{Le " plan global ॥ $^{34}$ et la statistique graphique}

La réflexion d'ensemble serait cependant incomplète si nous n'abordions pas la notion de carte générale et plus particulièrement les cartes issues de traitement statistiques - le traitement automatique de données y prend ses dimensions les plus évidentes. Ce type de cartes, très largement utilisé pour traduire les phénomènes économiques et sociaux, connaît des ancêtres prestigieux. Comment ne pas citer ici les remarquables productions du XIXe siècle, les travaux de Joseph Minard dans les années 1850 et les publications issues de la Statistique générale de la France comme les Albums de statistique graphique publiés à partir de $1879^{35}$. Les cartes liées aux transports, aux circulations des matières premières, à l'industrie y sont nombreuses.

En histoire des techniques et de l'industrie aujourd'hui, la carte thématique générale est assez régulièrement utilisée dans les publications d'ensemble sur un territoire donné. Citons les Indicateurs du patrimoine industriel, publiés par les différents services régionaux d'inventaire qui font ainsi presque systématiquement appel à ces cartes statistiques en cartographie numérique : typologie des unités étudiées bien entendu mais également régulièrement, éléments de datation et de chronologie des différentes installations industrielles ${ }^{36}$. Ce qui se dessine avec ces synthèses, c'est peu à peu la naissance de la compréhension de l'importance globale des usines dans les territoires, cette notion de paysage industriel que défendait si justement Maurice Daumas $^{37}$.C'est peu à peu également la notion

34 D'après l'expression employée dans Espace français. Vision et aménagement, $X \mathrm{Vl} \mathrm{l}^{\mathrm{e}} \mathrm{XI} \mathrm{X}^{\mathrm{e}}$ siècle, Paris, Archives nationales, 1987, p. 185.

35 Gilles Palsky, Des chiffres et des cartes : naissance et développement de la cartographie quantitative française au XIXe siècle, Paris, Comité des travaux historiques et scientifiques, 1996

36 Nathalie Van Bost, Brasseries et malteries (Nord-Pas-deCalais), Lille, La Voix du Nord, 2000, avec une belle analyse en quatre cartes de la densité des brasseries depuis 1888, pp. 18-19 ; Pascale Moisdon-Pouvreau, Patrimoine industriel des Deux-Sèvres, La Crèche, Geste éditions, 2005 (le nombre des usines actives de 1880 à 1950, confronté aux sites étudiés, pp. 10-11). On est cependant frustré par le principe de ces ouvrages qui interdit aux chercheurs de publier les plans des sites qui figurent pourtant régulièrement dans les dossiers des services régionaux d'inventaire du patrimoine. 37 Voir les travaux de Marina Gasnier qui a su composer sa thèse publiée sous le titre Le paysage de l'industrie en Ille-et-Vilaine, $X I X^{e}-X X^{e}$ siècles, Rennes, Presses universitaires de Rennes, 2003, à partir de ses travaux à l'inventaire général de Bretagne : id., Patrimoine industriel de l'llle-et-
d'Atlas qui émerge au sein de la communauté. Sans doute, les avancées en termes de connaissance du patrimoine industriel permettent-elles plus facilement les publications de ce genre ${ }^{38}$. Tout en posant la question patrimoniale, une publication toute récente à Lyon ouvre plus généralement sur le territoire de l'industrie, avec le souhait avoué de pouvoir disposer à terme d'un $\mathrm{SIG}^{39}$

L'étude peut cependant aller beaucoup plus loin. Nous citerons pour seul exemple le travail remarquable mené lors de l'enquête sur les forges du Maine ${ }^{40}$. Toutes les échelles des plans et des cartes SIG ont ici été mises en oeuvre au profit d'une histoire technique et industrielle : de l'usine, nous l'avons vu précédemment, aux nombreuses cartes régionales et jusqu'aux cartes statistiques nationales. Telle carte explicite les services dus pour les forges, telle autre le commerce du fer dans le Maine au XVIII siècle ou l'approvisionnement en bois ${ }^{41}$. Plus loin, passant à la France, on dispose des statistiques de production de fonte de seconde fusion pour la fin du XIXe siècle ou encore de la répartition de la clientèle Chappée entre 1882 et $1932^{42}$. On se situe dans cette tentative systémique de compréhension de la technique, dans cette histoire plus globale "qui a marqué les paysages, mobilisé hommes et capitaux $\|^{43}$.

\section{L'état de la recherche au Centre François Viète}

Le Centre François Viète à Nantes développe actuellement ses axes de recherche d'histoire des techniques autour de deux directions principales: le monde industrialo-portuaire et le littoral du point de vue thématique ${ }^{44}$, l'intégration des technologies

Vilaine, Paris, Éditions du patrimoine, 2002.

38 Gracia Dorel-Ferré, Atlas du patrimoine industriel de Champagne-Ardenne. Les racines de la modernité, Reims, CRDP de Champagne-Ardenne, 2005 ; Cécile Gouy-Gilbert, Jean-François Parent, Atlas du patrimoine industriel de I'Isère-Un état des lieux au début du XXIe siècle, Grenoble. Patrimoine en Isère, 2007.

39 Florence Berthet, Anne Gigolotti, Sarah Wasserstrom, Atlas de l'aventure industrielle de l'agglomération lyonnaise, Lyon, Agence d'urbanisme pour le développement de l'agglomération lyonnaise, 2009.

40 J.-F. Belhoste, É. Robineau et alii, La métallurgie du Maine, op.cit, cartographie de Christohe Batardy et infographie de Marc Brugier.

41 Ibid., pp. 111,228 et 255.

42 lbid., pp. $301,314$.

43 Denis Woronoff, «Préface », dans ibid., p. 15

44 Histoire et techniques du paysage industriel portuaire en France, $X V I^{e}-X X^{e}$ siècles, colloque organisé par le Centre François Viète d'Épistémologie, histoire des sciences et des techniques (EA 1161), Brest-Nantes, avril et juin 2008, sous

86 Documents pour l'histoire des techniques $\mathrm{n}^{\circ} 18$ - décembre 2009 
numériques à l'histoire du point vue méthodologique. À ce dernier titre, nous l'avons écrit plus haut, la collaboration étroite avec l'École centrale de Nantes est fondamentale dans notre dispositif 45

Nos recherches personnelles s'organisent en ce moment autour de deux directions plus précises : l'étude des chantiers navals de La Ciotat et la rétroconception d'une machine ancienne sur les modèles déjà éprouvés dans le laboratoire, modélisation d'un grand plan relief du port de Nantes selon les mêmes méthodologies mais avec une complexité et des enjeux plus développés.

La première thématique correspond à la modélisation d'une cintreuse horizontale des années 1950 à La Ciotat - démolie depuis - à la suite d'une première étude commandée par la Direction régionale des affaires culturelles (DRAC) de ProvenceAlpes-Côte d'Azur (PACA) consacrée à une mise au point sur l'histoire générale du site des anciens chantiers navals et son évaluation patrimoniale, en particulier pour les engins de levage ${ }^{46}$. La modélisation et l'étude du contexte technique et industriel de la machine mobilisent également les compétences d'un maître de conférences et d'un élève-ingénieur de l'École centrale de Nantes ${ }^{47}$. L'objet de la recherche actuelle serait de préciser les différents fonctionnements des machines dans l'ensemble du procédé industriel. Nous n'en sommes pas encore à la modélisation des circuits de production, c'est-àdire à la véritable ambition de l'analyse spatiale, mais l'intégration virtuelle d'un objet comme la cintreuse dans l'ensemble du territoire de l'usine nous parait bien sûr constituer une étape nécessaire. La première phase de l'étude avait en effet été l'occasion

la direction de Jean-Louis Kerouanton et Sylvain Laubé (à paraître) ; Objets et territoires maritimes et navals : histoire et patrimoine, journée d'étude, Nantes, Musée du château des ducs de Bretagne, juin 2009. Le Centre François Viète est membre du GIS d'Histoire maritime depuis 2008.

$45 \mathrm{~F}$. Laroche, op. cit. Notre collaboration passe par une convention de partenariat IRCCYN - UMR 6597/Centre François Viète. 46 Jean-Louis Kerouanton, Les chantiers navals de la Ciotat, rapport de recherche, Centre François Viète, université de Nantes, DRAC Provence-Alpes-Côte d'Azur, décembre 2007. 47 Résultant d'un nouveau contrat de recherche avec la Direction régionale des affaires culturelles Provence-AlpesCôte d'Azur, ce travail a fait l'objet d'une première communication par Jean-Louis Kerouanton, Didier Serveille, "La cintreuse à membrures Bennie et les chantiers navals de la Ciotat ", Objets et territoires maritimes et navals: histoire et patrimoine, Nantes, Musée du château des ducs de Bretagne, juin 2009 et d'une courte restitution vidéo par Jean-Louis Kerouanton, Florent Laroche, Didier Serveille, La cintreuse. Cinématique. d'utiliser les outils SIG pour établir les plans généraux d'implantation à différentes dates. Nous avons pour cela utilisé des plans anciens disponibles que nous avons géoréférencés ${ }^{48}$. Cette étape permet ensuite de superposer automatiquement les différentes informations chronologiques sans problème d'échelle et d'orientation. La simple superposition visuelle de plans de différentes natures selon ce procédé est déjà très éclairante. Elle l'est davantage encore quand on redessine les différents plans sous format vectoriel. Les différentes informations graphiques peuvent ainsi être transformées en polygones, en lignes ou en points auxquels on peut rattacher toutes les informations nécessaires organisées en base de données. Le plan des chantiers de $1952^{49}$ va nous servir d'exemple. Ses mises en rapport possible avec les informations plus récentes informent considérablement l'évolution du site dans une phase fondamentale de restructuration industrielle : extensions progressives sur le domaine public maritime, réorganisation globale des chantiers, passage d'un circuit de production aux éléments plus éloignés des cales de construction à une rationalisation du « nouveau circuit ». Les machines encore en place au moment de l'étude sont également localisées et informées dans le dispositif.

La deuxième recherche évoquée est une commande du Musée du Château des Ducs de Bretagne au Centre François Viète ${ }^{50}$. La modélisation de la maquette du Port de Nantes de 1900 (un grand plan relief au $1 / 500^{\circ}$ environ, de $16 \mathrm{~m}^{2}$ ) met en œuvre à des fins de recherche et de valorisation grand public un ensemble de compétences particulièrement vaste ${ }^{51}$.

48 Le géoréférencement est le rattachement de données à des coordonnées géographiques. On peut géoréférencer des données purement graphiques, comme des images préexistantes de cartes ou de plans, mais aussi des données vectorielles, comme des plans d'architecture conçus numériquement (sous AutoCAD par exemple). Dans le même système de projection, toutes ces données seront cohérentes entre elles et sont donc comparables et superposables. Les images anciennes, opaques par définition, peuvent désormais sur certains outils être observées en transparence plus ou moins grande ; c'est par exemple le cas sur le site public géoportail (www.geoportail.fr) où les cartes topographiques sont plus ou moins visibles sur fond de photographies aériennes.

49 Archives SEMIDEP- La Ciotat.

50 Responsable scientifique, Jean-Louis Kerouanton ; comité de pilotage, Jean-Louis Kerouanton, Florent Laroche (ECN Nantes/ IRCCyN), Bertrand Guillet et Christophe Courtin (Musée d'Histoire du château des ducs de Bretagne).

51 Florent Laroche, Christophe Courtin, « La maquette du port de Nantes : modélisation d'un territoire technique ", Objets et territoires maritimes et navals : histoire et patrimoine, Nantes, Musée 
Il s'agit en effet de considérer l'objet pour lui-même comme pièce de musée mais aussi comme élément documentaire majeur pour la compréhension de Nantesen $1900^{52}$. Pourl'historien destechniques, ils'agit de comprendre et d'expliciter les développements industriels et portuaires dans leur ampleur spatiale et territoriale, les fonctionnements internes des usines, mais aussi leurs connexions. Le projet s'étend sur trois ans (2008-2011) : à partir de la numérisation de la maquette, on obtiendra une «photographie »3 $3 \mathrm{D}$ de l'objet, informée sous forme de base de données, qui sera ensuite restituée, à plusieurs niveaux, en réalité virtuelle auprès du public et des chercheurs. L'équipe constituée pour ce projet réunit des compétences d'enseignants-chercheurs et d'élèves de l'Université de Nantes (historiens, informaticiens, électroniciens) et de l'École centrale de Nantes (mécaniciens, spécialiste de génie industriel) ; elle devrait s'étendre pour les phases suivantes à des géographes et à des architectes. C'est l'ensemble d'un territoire donné qui est interrogé dans sa complexité urbaine et portuaire. L'objet lui-même a d'ailleurs évolué. Présenté en 1900 à l'Exposition universelle dans le cadre du pavillon des Chambres de commerce portuaires, il a ensuite servi de "démonstrateur " permanent dans le hall de la Chambre de commerce de Nantes, jusqu'en 1914. ॥ a ainsi connu des mises à jour régulières : comblement d'un canal, mise en place du pont transbordeur lors de sa construction en 1903, établissement progressif des quais... La démarche historienne consistera d'abord à critiquer l'objet lui-même avant d'en tirer les informations possibles pour l'histoire nantaise.

du château des ducs de Bretagne, juin 2009, communication. 52 Nous avions déjà collaboré avec le Musée du château à propos de cet objet important. Bertrand Guillet, Laurent Huron, Jean-Lovis Kerouanton, "La maquette du Port de Nantes $11,124^{e}$ congrès national des sociétés savantes, "Des villes, des ports, la mer, les hommes" (Nantes, 1999), Paris, CTHS, 2001, pp. 143-155.

\section{Conclusion}

Nous sommes bien conscients que l'essentiel du cheminresteàfaire. On peutimaginerprogressivement pouvoir « rentrer» dans les ateliers eux-mêmes par un simple changement d'échelle, mais à la condition de disposer de la documentation adéquate. Un des avantages importants de la "navigation " SIG est en effet cette possibilité permanente de changer les échelles de lecture et cette réflexivité du plus petit au plus grand. Encore faut-il imaginer ce système conceptuel que doit être le SIG. On aura compris que la question interdisciplinaire est au cœur de la démarche méthodologique que nous essayons d'initier; elle ne pourra se développer dans ce domaine précis sans la collaboration de cartographes, sans contact et sans dialogue avec la géographie ${ }^{53}$.

Une des voies d'avenir en termes de SIG dépasse déjà l'élaboration des données brutes et leur mise en forme systémique par les chercheurs. Les accès sur internetse développentaujourd'huiàgrande vitesse. Certains sites permettent désormais la juxtaposition de leurs propres données avec des importations personnalisées si elles sont déjà géoréférencées ${ }^{54}$. C'est probablement une des pistes vers laquelle doivent se tourner également l'ensemble de nos recherches en termes d'inter-opérabilité : la mise en ligne espérée des modélisations dynamiques des machines anciennes, intégrées du point de vue géographique aux données spatiales du paysage technique et industriel.

53 Une des premières pierres à ce dialogue nécessaire a été posée, cheznous, grâceà la contribution de la géographe, Nacima Baron, « Pour ouvrir le débat, regard d'une géographe : histoire des techniques, espace et territoire ", Objets et territoires maritimes et navals : histoire et patrimoine, Nantes, Musée du château des ducs de Bretagne, juin 2009, communication. 54 C'est déjà le cas du site piloté par le BRGM [http://infoterre.brgm.fr] qui permet l'import de données spatiales supplémentaires par le biais de protocoles spécifiques (WMC, Web map context, piloté par l'OGC, Open geospatial consortium) pour des données en XML qui préservent le géoréférencement. Cette opération est également possible sur Google Earth. Elle le sera bientôt sur certains sites locaux : le conseil général de la Seine-Saint-denis est en pointe sur ce sujet avec des liens ouverts qui devraient être opérationnels début 2010 sur l'ensemble du site cartographique du département dont l'atlas du patrimoine qui est déjà en ligne [ www.atlas-patrimoine93.fr ]. 


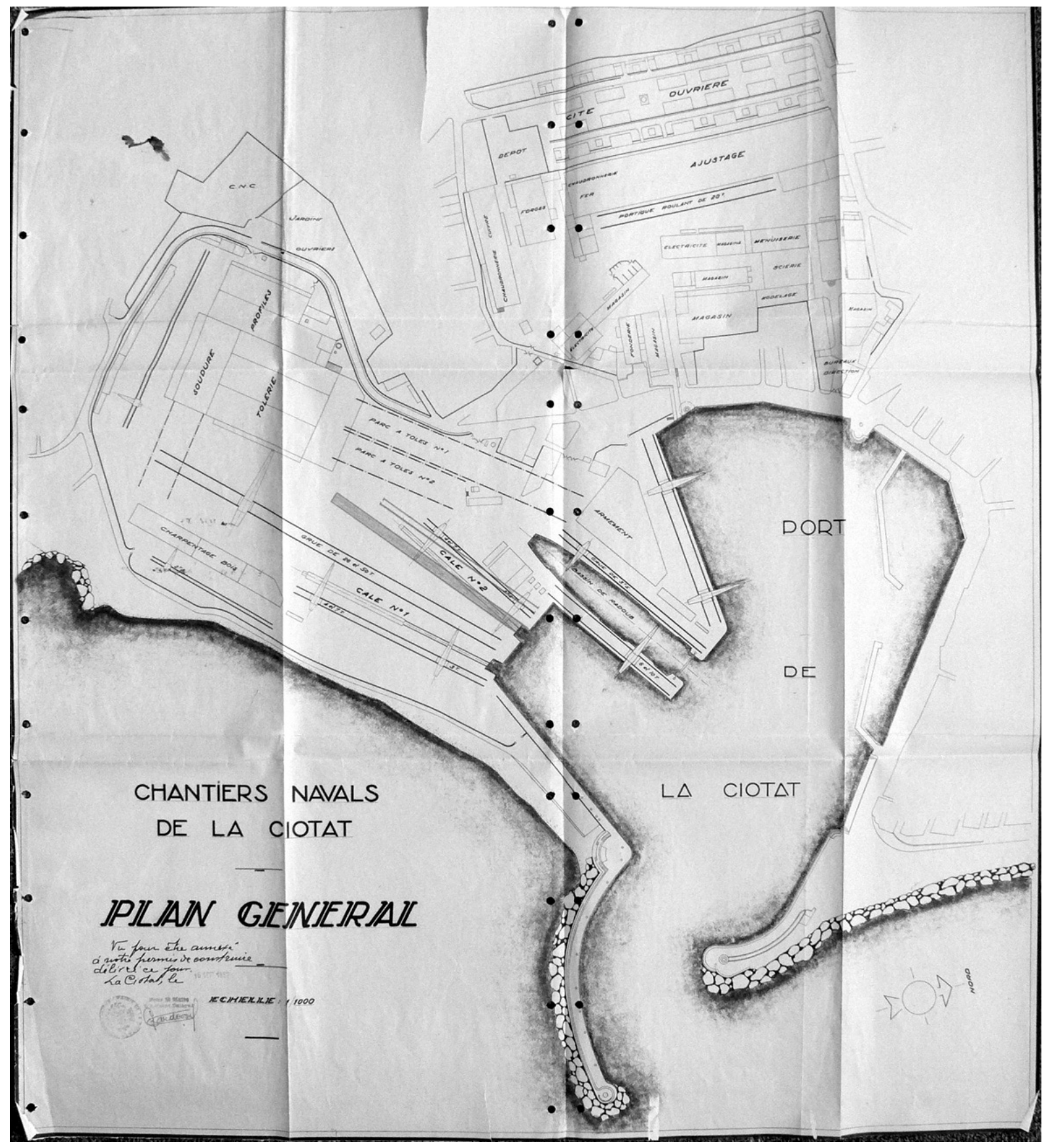

fig. 1 - Chantiers navals de La Ciotat, 1952, Archives SEMIDEP2009 (cliché Jean-Lovis Kerouanton) 


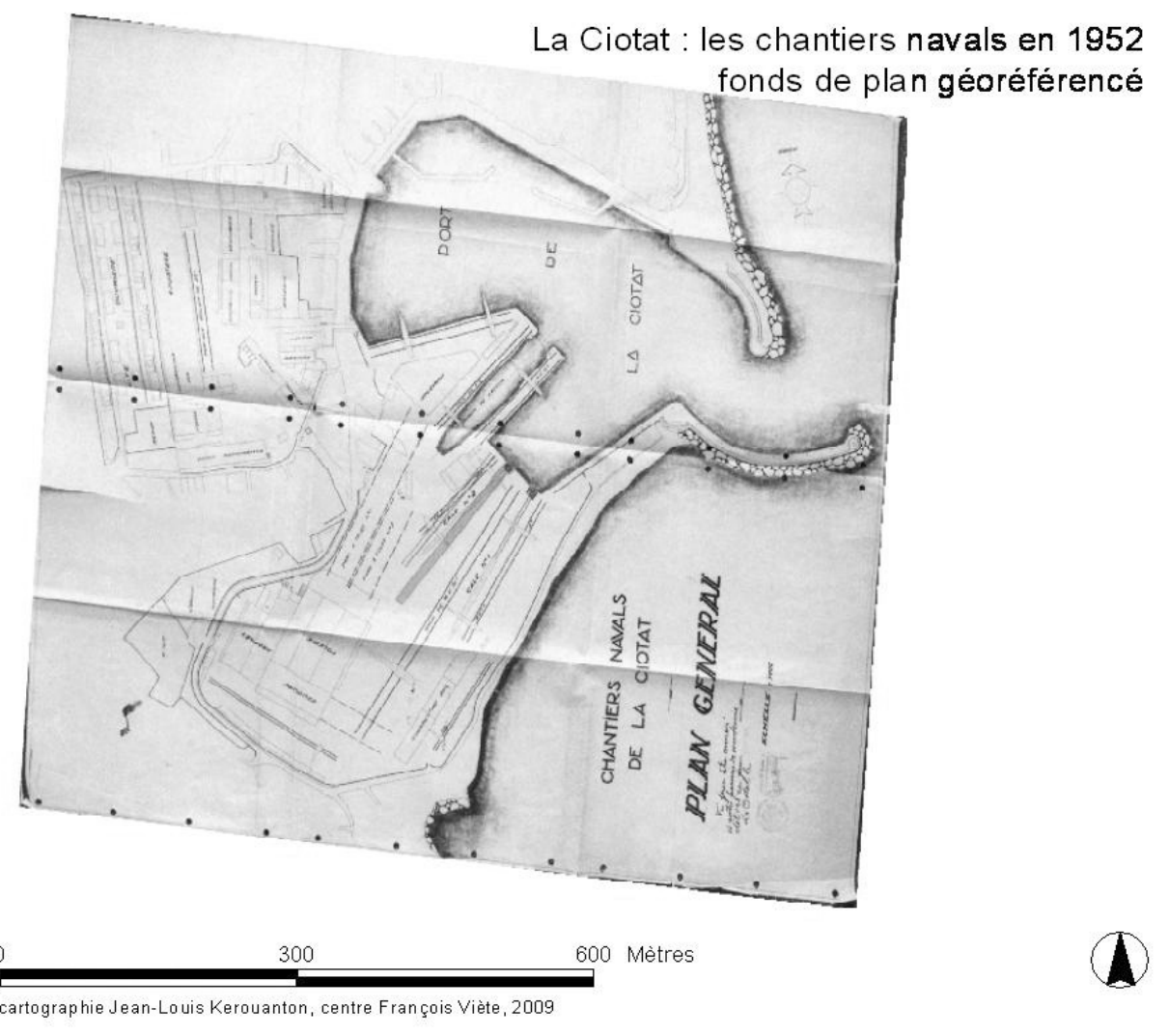

fig. 2 - Géoréférencement du plan de 1952 (cartographie Jean-Louis Kerouanton, 2009).

fig. 3 - Interprétation vectorielle du plan de 1952 (cartographie Jean-Louis Kerouanton, 2009).

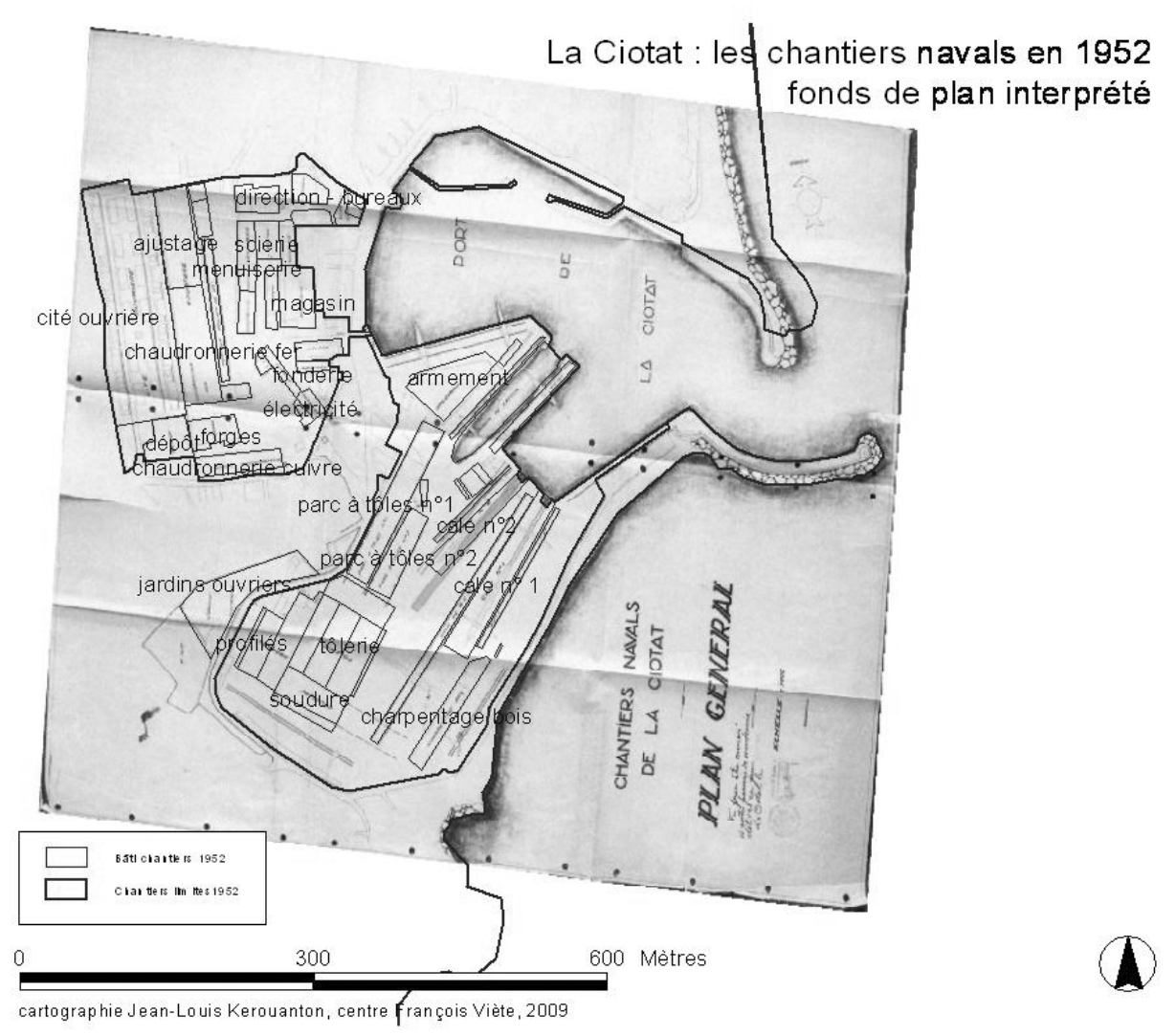

$90 \diamond$ Documents pour l'histoire des techniques $\mathrm{n}^{\circ} 18$ - décembre 2009 


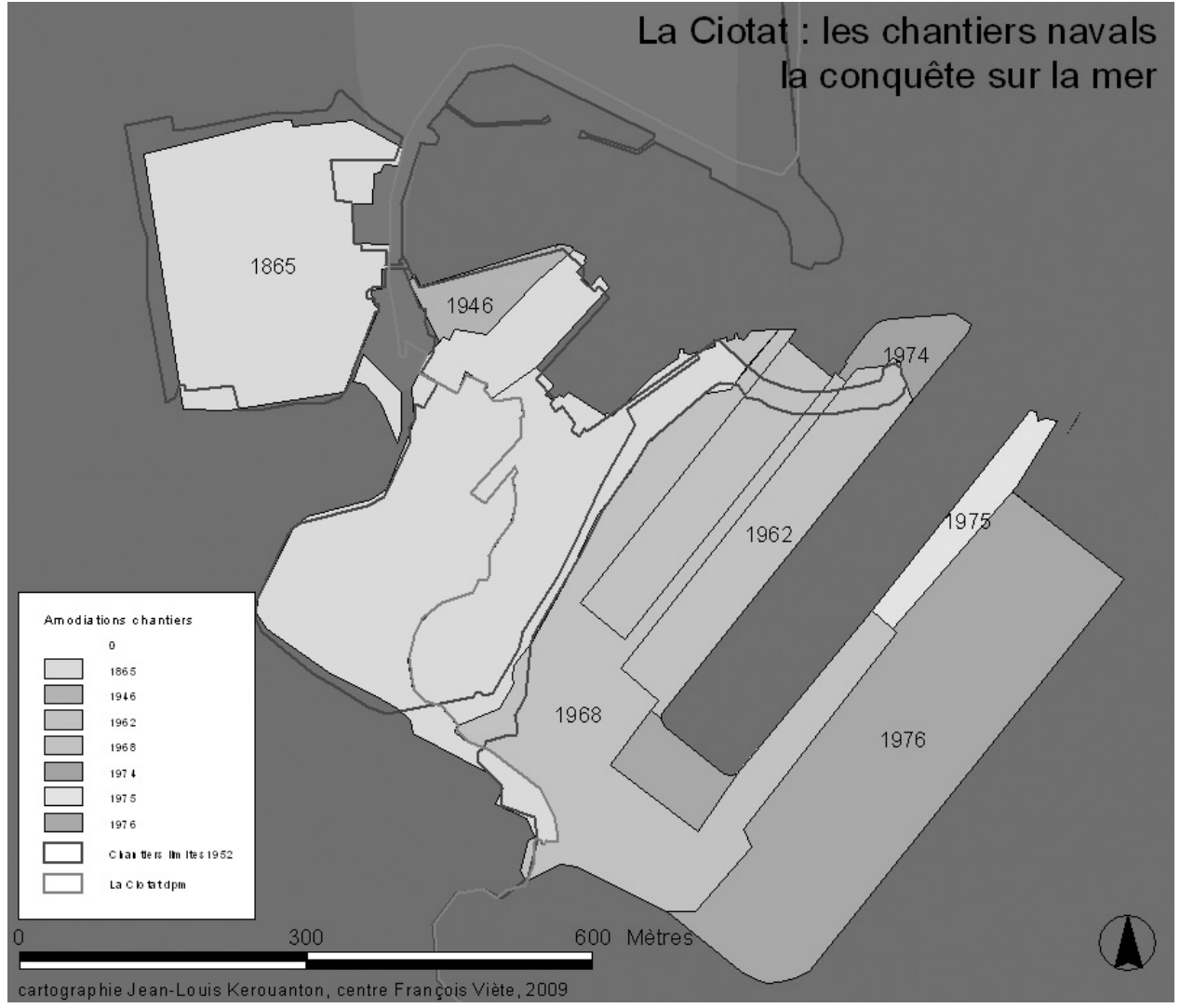

fig. 4 - Des informations superposées : I'évolution des

chantiers (cartographie Jean-Lovis Kerouanton, 2009).

fig. 5 - Implantation générale et emplacement des machines restantes en 2007 (cartographie Jean-Lovis Kerouanton, 2009).

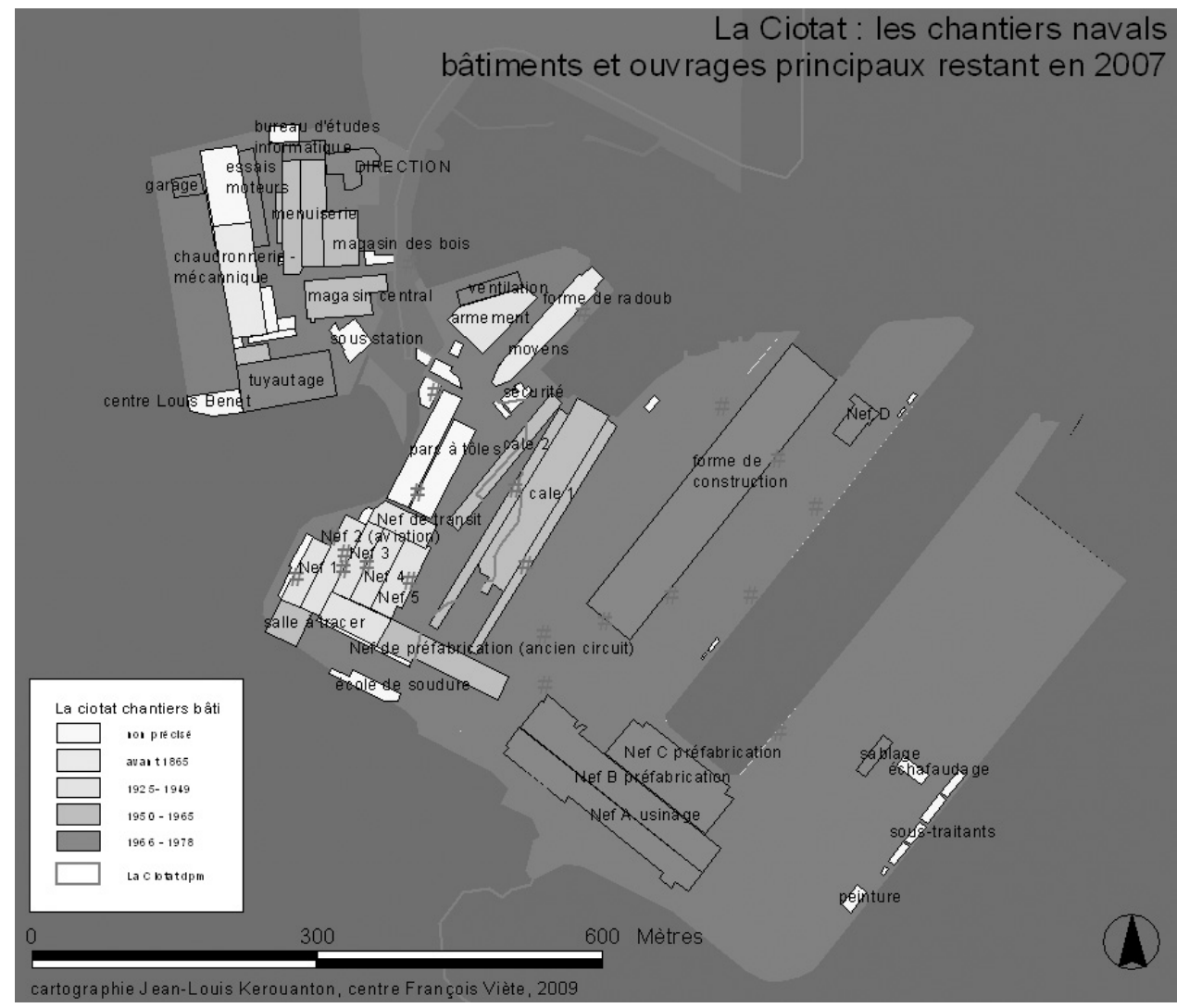




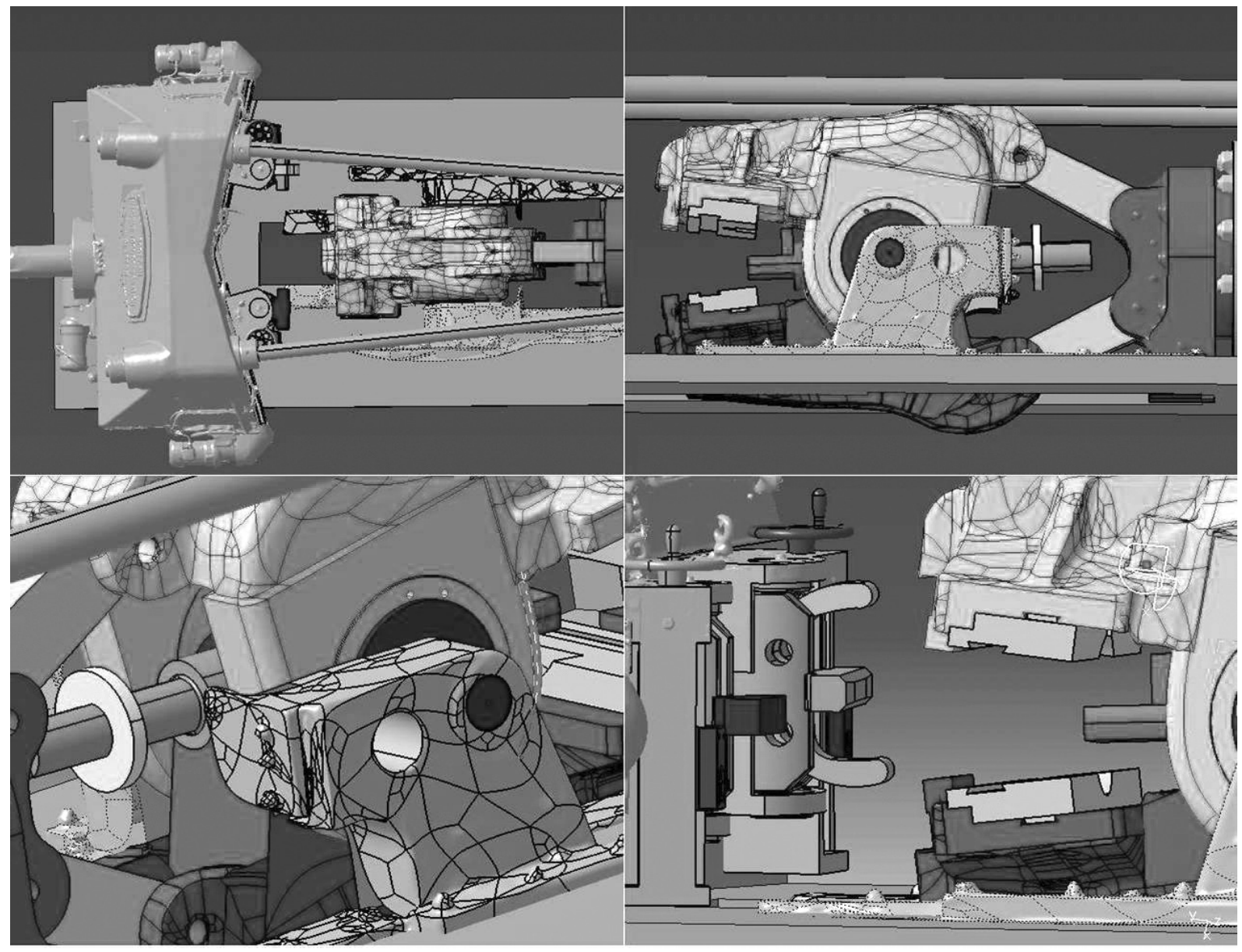

fig. 6 - Modélisation de la cintreuse à membrures Bennie, détails (modélisation Didier Serveille, 2009).

fig. 7 - Vue de la maquette du port de Nantes lors de la numérisation, juin 2009 (cliché Jean-Lovis Kerouanton).

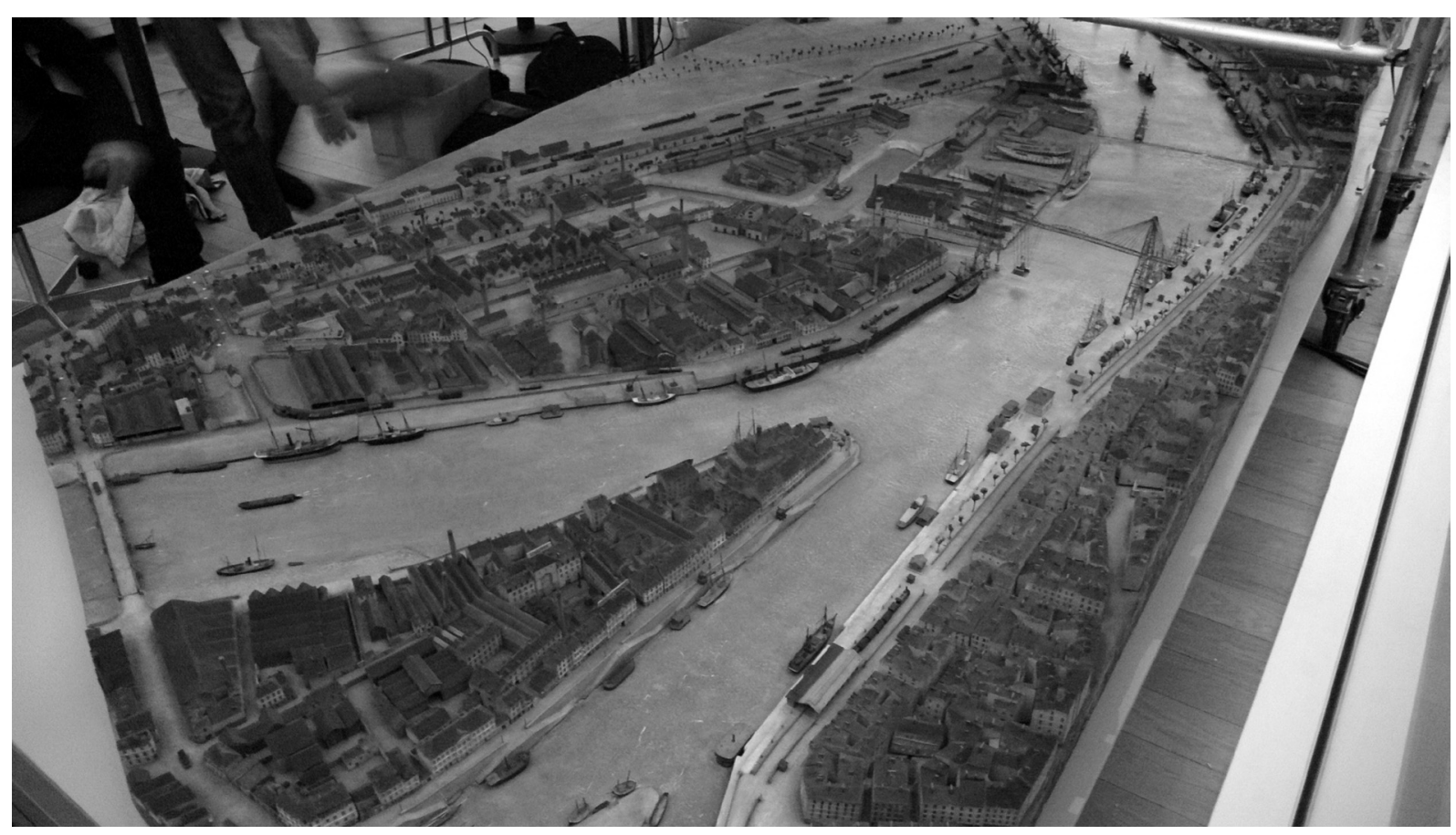

$92 \downarrow$ Documents pour l'histoire des techniques $\mathrm{n}^{\circ} 18$ - décembre 2009 


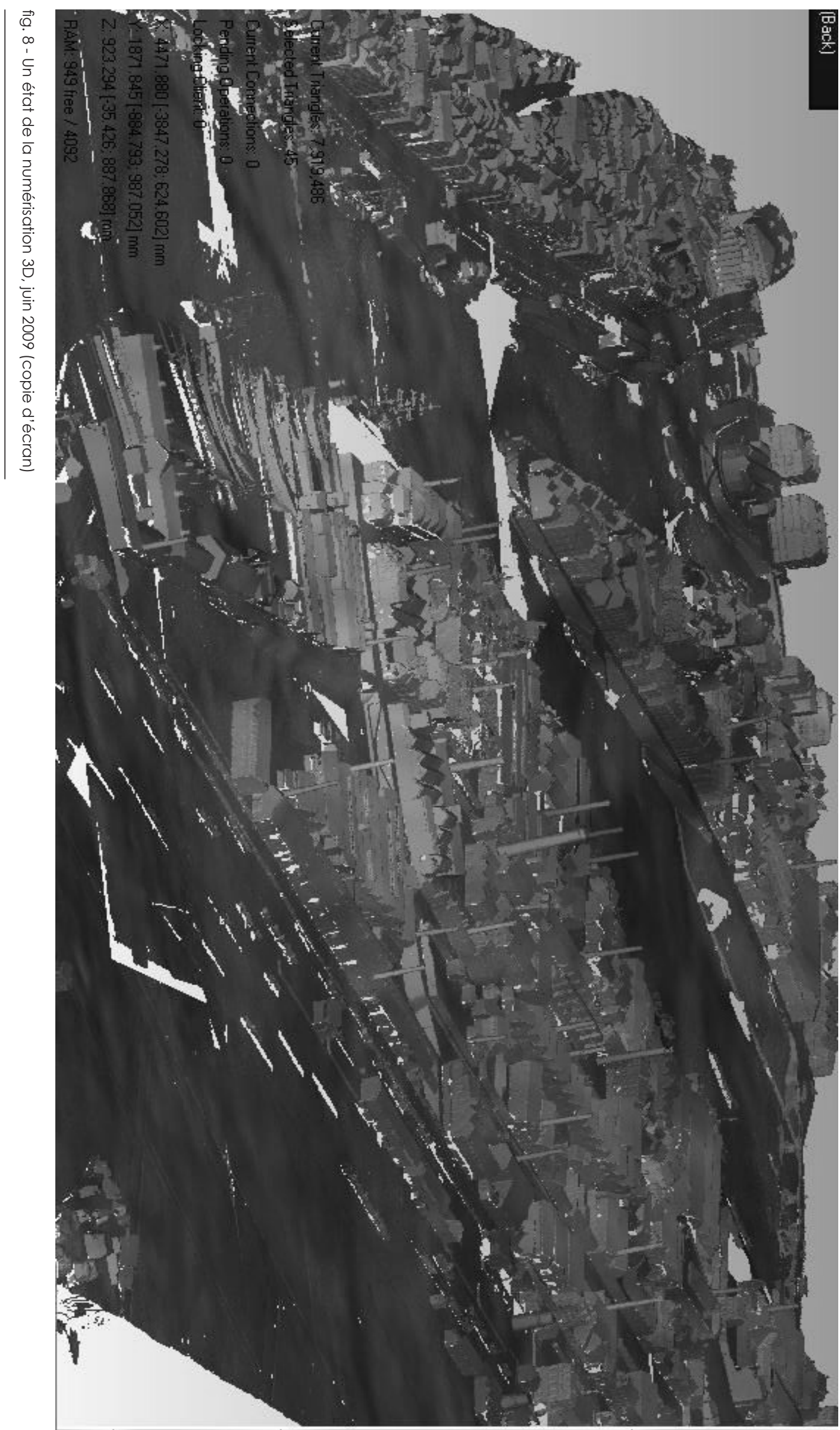




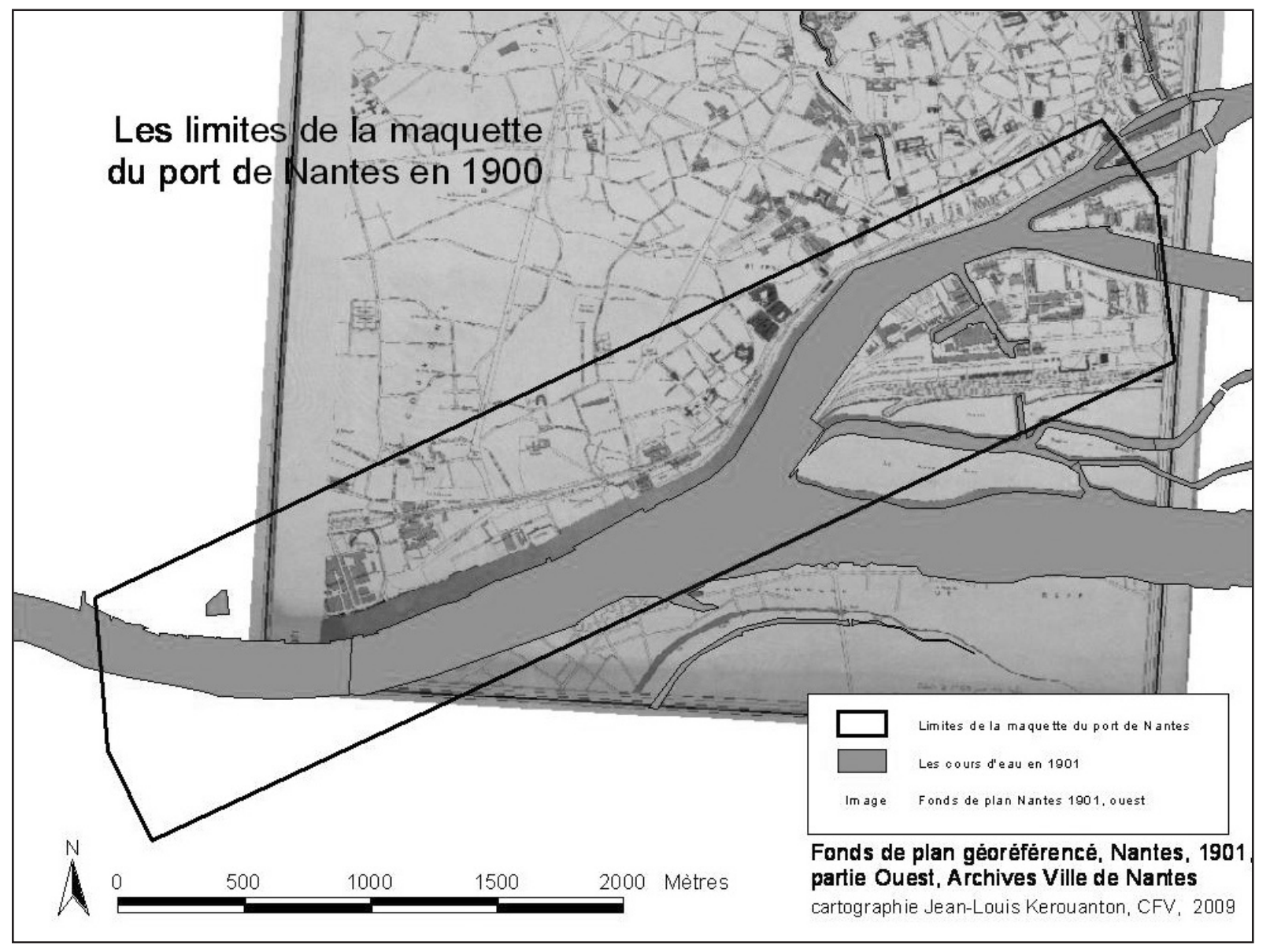

fig. 9 - L'emprise de la maquette par rapport aux plans anciens géoréférencés (cartographie Jean-Lovis Kerouanton, 2009). 